\title{
Sustainable mobility - analysis of sustainable mobility measures in cities
}

\author{
N. L. Sá ${ }^{1,2}$ \& J. B. Gouveia ${ }^{3}$ \\ ${ }^{1}$ Department of Environmental Health, \\ College of Health Technologies of Coimbra, Portugal \\ ${ }^{2}$ Sustainable Energy Systems, University of Aveiro, Portugal \\ ${ }^{3}$ GOVCOP and Department of Economy, Management and Industrial \\ Engineering, University of Aveiro, Portugal
}

\begin{abstract}
Mobility is assumed as a sine qua non condition on people's relationships. However, the raising of mobility dependence on the existing energy paradigm has been hazardous to the environment, to the economy and, consequently, to society. In this context began a great necessity to rethink all mobility paradigms and change into more efficient and sustainable cities, in order to preserve present society needs without compromising future generations. One of the most valuable tools used in recent years is the Sustainable Mobility Plans, which have been adopted in several European cities. These are expected to be an efficient measure in achieving the European goals, namely the reduction of $\mathrm{CO}_{2}$ emissions, one of the main causes of Climate Change. This work shows the state of the art of Sustainable Mobility Plans in three territories, as a means to achieve more Sustainable Cities in the near future. The main conclusions of this review are the absence of a standard indicator to measure the mobility in cities, which is as an important issue to develop; the necessity of integrated pull and push measures in cities as a way to change people's behaviours and the importance of a city's network on the best practices in mobility and urban planning, to achieve the "Sustainable City" goal.
\end{abstract}

Keywords: sustainable mobility, mobility plans, energy, transports. 


\section{Introduction}

The current energy paradigm, the notion of sustainability and mobility, forced the European Union to take action on policies regarding the spatial, social and environmental context on sustainable cities, as well as on people and goods mobility and their eco-spatial relationships. The sustainable mobility plans of three European cities have been studied in order to understand the adopted measures in each one and to build a critical analysis.

\section{Energy policy at the European Union}

The trans-European transport networks and, in general, the efficient services on transport are crucial to the urban development and policies. Especially a good transport system is a determinant factor on the urban economy competitiveness and in the quality of life of citizens. The concept of sustainable mobility became the main goal on the common transport policy, which intends to reconcile the mobility demand by individual and economical activities with the recognition of the resources limits and the impacts of these transport operations on the environment. The common transport policy also aims to address questions as the integration of the priorities of space planning in the arrangement of transport infrastructures and in the promotion of intermodal transportation, without compromising social, economic and environmental aspects [1].

In 1996 it was elaborated by the European Commission in the Green Book "The Citizen's Network: Fulfilling the Potential of Public Passenger Transport in Europe". This document displayed the advantages of the public transports and the main action areas at a communitarian level destined to stimulate and promote an integrated system of intermodal transports that explores entirely the potential of public transportation. This paper came to encase other documents of the European Commission, as the Green Book (1990) about urban environment and the Report on the Sustainable Cities (1996) on the promotion of an integrated board of the urban problems. The Sustainable Cities project was initiated in 1993, aiming to stimulate cities to establish and to apply the Local Agenda 21 or similar Sustainability Plans, based on policies reports, interchange of experiences, networks and diffusion of the best practices cases, which are still in use.

It is easily understood that the guarantee of fastest mobility of people and merchandises, efficiently and at low cost it is fundamental for the economy dynamism and society cohesion of the EU. The transport sector generates $10 \%$ wealth of the EU in terms of gross internal product (GIP) and assures more than ten million jobs. However, the constant increase of mobility submits the systems of transports to great pressure, giving origin to congestions in what road and aerial traffic are concerned, reducing the economical effectiveness and contributing for an increase of fuel consumption and pollution.

One of the main issues is the fact that the transports sector, mainly the road transportation, is responsible for $28 \%$ of $\mathrm{CO}_{2}$ emissions, the main gas with greenhouse effect, in the EU. Hence, it was introduced measures to promote 
more efficient and alternative fuels utilization, in order to reduce the carbon emissions of vehicles.

In 2001, the European Commission elaborated an action plan for the transport sector for ten years - "White Paper - European transport policy for 2010: time to decide". According to a intercalate evaluation carried through in 2006, the plan will have to be centred in the improvement of the competitiveness of the rail ways, in the introduction of one maritime policy, in the development of transport systems which use the best technologies available, in the application of taxes for the infrastructure usage, in the increase of biofuels production and in the paths to reduce the congestion in the cities.

In October of 2007 the European Commission presented the "Green Book Towards a new culture of urban mobility" that became incipient in the solutions and in the necessary strategies, until this date. This policy came to be strengthened, recently, with the "Green Paper - Revision of the relative policy to the RTE-T" for a better integration of the trans-European transports network, at the service of the common policy of transports, which gave an accurate reply after the White Paper launched in 2001 with goals up to 2010.

At the same time the European Union launched the Directive 2003/30/CE in May of 2003, related to the promotion of the use of biofuels or other renewable fuels in transports. Due to this policy, there was a significant increase of this type of fuel utilization in the transports sector, particularly in road transportation. State-Members will have to take measures to assure the substitution of 5,57\% of all the fossil fuels (gasoline and diesel) used for the purpose of transport for biofuels, up to 2010 , beyond the directive $32 / 2006 / \mathrm{CE}$ that defines an energy efficiency plan in the final use of energy and to the energy services.

In 2008 the president of the European commission, in the scope of climate changes, presented a communication (COM (2008) 30), where he described the so called " $20-20-20$ by 2020 ", where he considers the increment of $20 \%$ of renewable energy, the increase of $20 \%$ of the energy efficiency and the reduction of $20 \%$ of the $\mathrm{CO}_{2}$ emissions, all up to 2020 [2].

\section{Energy, sustainability and mobility}

As the transport system is in the foundations of the progress in modern societies, the sustainable development demands a sustainable transport system. Among many definitions that had been suggested for the concept of sustainable mobility, one is distinguished, which was proposed in the Mobility 2030 report: "Meeting the Challenges to Sustainability" from the World Business Council for Sustainable Development (WBCSD [3]):

"Sustainable Mobility not only requires that essential human or ecological values not be sacrificed today or in the future. It also requires that society's needs to move freely, gain access, communicate, trade, and establish relationships be met. The goals are intended to ensure that mobility continues to fulfil its indispensable role in improving the living standards of the global population by reducing disparities in mobility opportunities between and within 
countries and by providing enhanced mobility alternatives to the general populations of countries in both the developed and developing worlds" [3].

There are three identified dimensions of sustainable mobility in the previous definition that had been described by the OCDE/AIE (2001):

- Mobility and Accessibility Dimension: to offer alternatives of accessibility and transport services adequate and available to meet the necessities of mobility of people and goods;

- Social dimension: to offer adequate transport services to all elements of society in order to not compromise its stability, namely in safety, health, congestion and equity in the access to these services, for the different segments of the population. Thus, the system must guarantee the adequate level of territory coverage in the net drawing and in the schedules of the transport systems, as well as in the obligations of a public services in transports, specifically in what regularity, capacity and the accessible tariffs of these services is concerned;

- Environmental dimension: to offer transport services in order to not degradate the environment or to not compromise the possibility of citizens in getting other essential resources for their regular subsistence or to develop other activities with these same resources [4].

Beyond mobility and accessibility, social and environmental equity dimensions, a sustainable system of transports cannot be dismissed from the economical efficiency goal. This is equally essential for both production of the transport services and consumers. The achievement of these goals in any of the dimensions is not opposite to the search of economical efficiency, but it must be integrated in it [5].

In light of what was mentioned above, one highlights the adequate relationship between Energy, Sustainability and Mobility, which is fundamental due to:

- Urban transport is nowadays responsible for about $40 \%$ of total energy consumption in the European Union and for $40 \%$ of $\mathrm{CO}_{2}$ emissions, accountable for the climate change, as other pollutants, being also liable for the degradation of life conditions of its inhabitants;

- The urban trips, which average travel distance is less than $15 \mathrm{~km}$, are responsible for one fifth of the total kilometres travelled in all Europe and the trips with a travel distance less than $10 \mathrm{~km}$ are to blame for three quarters of the same total;

- The urban dispersion is increasing the average distance of urban trips. Between 1995 and 2030, it is estimated that the kilometres to be travelled in urban areas will rise $40 \%$. The sub-urbanization and the reduction of urban density, the increasing of car possession and the expansion of roads networks made the private car the main transport in the urban field, with a share of $75 \%$ of travelled kilometres in that mean of transport in the European Union;

The Kyoto protocol perspectives a cut of $8 \%$ of total emissions of $\mathrm{CO}_{2}$ between 2008 and 2012, relatively to 1990 levels, however with the current tendency, the $\mathrm{CO}_{2}$ emission levels will suffer an increasing of $40 \%$ in 2010 relatively to 1990 , just because of the transport sector. 
To face the referred problems, new conventions and associations emerged in Europe, such as Energie-Cités, Covenant of Mayors, as means to promote sustainable mobility and to increase energy efficiency in cities, with Sustainable Mobility Plans as we will see further on [6].

\section{The transport and the mobility}

Throughout time, the growth of productive capacities has created increasing necessities of mobility. However, is equally truth that technological advances linked to transport sector have harnessed structurally the economy's growth. In the $20^{\text {th }}$ century, the increasing of the transport sector was developed mainly within the petrol paradigm, because it was seen as cheap and inexhaustible, promoting the diffusion of cars and its massive proliferation. This reality influenced the population forms of organizing the economical activities, both in time and in space. The industrial, the commercial localization and the residential neighbourhoods localizations were done without thinking in the amplitude of the impacts of these policies.

Finally, when faced to the necessity to find a development model, as referred at the Brundtland Report, "that meets the needs of the present generation without compromising the ability of future generations to meet their own needs", the economical agents woke up for the inevitability to consider the transport impacts and consequently to reduce or mitigate de main costs that are associated [7].

These conclusions have emerged a reflection about the alternatives and the creation of a conscience, which translates a transformation of the urban agglomerates. This must be reflected upon and planned in order to answer to the energy efficiency requirement as much as possible in people's and goods transportation, as well as in urban planning. This triangle assumes a key importance in territorial energy planning, in order to meet the challenges of sustainability without compromising people's daily life.

This trinomial reflects the emergent discussion about sustainable cities, in order to face the current model of European cities, which consumes $75 \%$ of total energy consumption in the EU [6].

\section{Cities and the mobility}

On behalf of the publication of the Green Paper about Urban Environment, the European Commission created in 1991 a specialist group on Urban Environment with national representatives and other independent experts. Their main contributions are in latu sensu:

- to study how future strategies of urban planning and land utilization could integrate environmental goals;

- to advice the Commission about the way as the urban environment dimension could be developed in scope of the community environmental policies;

- to analyze how the Commission could contribute more efficiently to improve the urban environment. 
In 1993, recognizing the extension of the environmental problem, this expert group launched the "Sustainable Cities" Project, to be developed between 1993 and 1995 with the following goals:

- To contribute to the development and discussion about sustainability of European urban environments;

- To encourage an extended experience exchange;

- To promote the best sustainable practices locally and in long term;

- To formulate recommendations in order to influence de EU and State-members policies, including at regional and local levels, according to the resolution of Ministers Council of 1991.

The "European Sustainable Cities" report is one of the main results of the "Sustainable Cities" Project and it is considered to be the main support document of the second European Conference of Sustainable Cities, carried through in Lisbon - Portugal in October 1996. Other results of the "Sustainable Cities" project include the "Good practices guide" and the "European System about good practices information", useful summaries destined to specific groups and conferences adapted to the necessities of each State-member. The exchange of information and experiences is being encouraged by the European Campaign of Sustainable Cities, launched in May of 1994, during the first European Conference of Sustainable Cities carried through in Aalborg - Denmark. Besides the "associated networks", including the Municipal and Regional Councils of Europe (CCRE), International Council for Local Environmental Initiatives (ICLEI), the World Federation of Twin-Cities (WFTC), the World Health Organization (WHO) and Eurocités are actively pledged to share information and experiences between cities and in improving their consulting skills, based on experimental projects at local level demonstration [8].

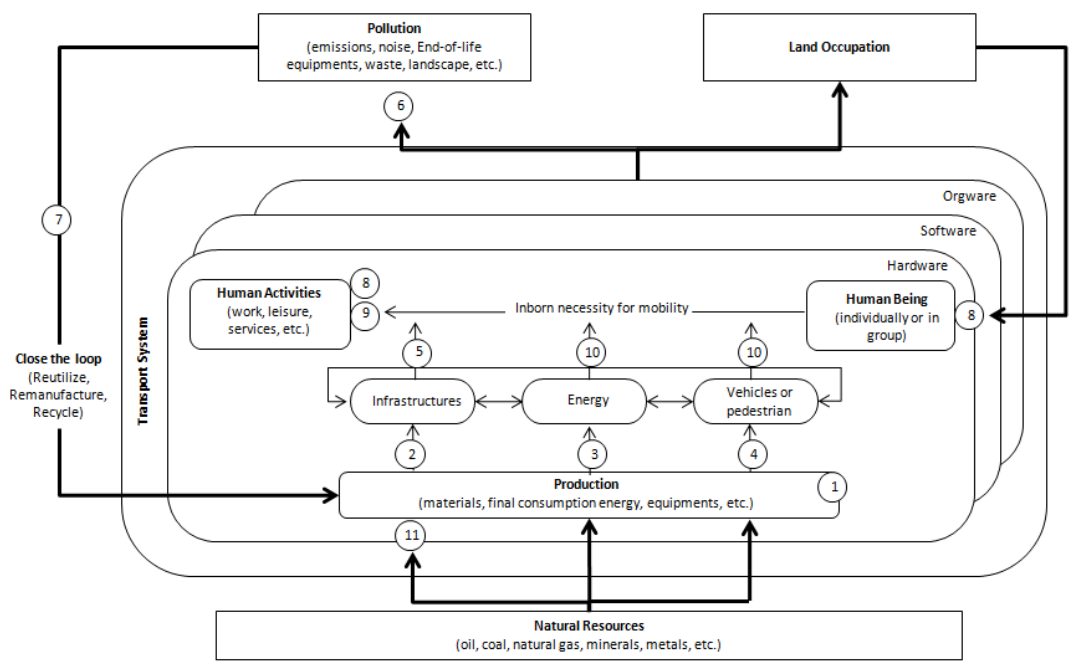

Figure 1: Possible interventions in the transport system to reduce environmental pressures [7]. 
As result of the studies obtained on this field over the last years, one concludes that the sustainable mobility consists in a global process developed in order to achieve a less intensive system of nature resources usage and pollution production. Due to this fact, decisions and actions are required as means to reduce the number of travels, encouraging a shift for more efficient technologies and to its environmental friendly utilization. The following chart summarizes the transport system.

Regarding the figure above, five main ideas are presented which will develop and explain this system:

1. Increasing the efficiency of the initial productive transport systems, namely the industrial process related to material production and building infrastructures of transport system support (1) and promoting reutilization, recycling and end-of-life equipments remanufacturing policies (7).

2. Improving vehicles efficiency:

a. Reduction of the specific consumption of vehicles (10);

b. Diminution of the emissions through systems of end-line treatment (for example, particle catalysis and filters) (6);

c. Transformation of travel characteristics for more efficient technologies, in the energy and environmental perspective (in application of transport policies, that is to say through tax incentives and disincentives): in the case of vehicles, for more efficient technologies of traction (for example, vehicles of electric traction) (4); concerning power plants, for alternative fuels (for example, GNC), electricity and hydrogen. One should retain that all factors must analyzed from the life cycle perspective (3).

3. Enhancing the efficient use of vehicles:

a. Economical techniques in driving (Eco-driving) and using information and communication technologies (ICT's) for a better performance in driving vehicles (for example, through On-Board Diagnostic systems) (10);

b. Better infrastructures design and engineering (specifically in pavements) (2) or;

c. Using ICT's to optimize the infrastructures utilization (for example, "Intermittent Bus Lane" technology which optimizes the utility and land efficiency of Bus Lanes, switching its status between private or both uses depending on proximity/presence or absence, respectively; or also a vehicles flux regularization, such as the ramp metering) (5).

4. Modal transfer and increase the rate of vehicle occupancy:

a. Reduction of individual transport using road pricing (Tax for moving vehicle phase and not only in static phase, as car parking) and more restrictive parking policies inside the cities (9); 
b. Incentive the use of the public transport, improving, among others aspects, the inter-modality through a bigger integration in some means and services of transportation;

c. Increasing vehicles occupancy in the main populations accumulations, through incentives such as High Occupancy Vehicles (HOV) lanes that could change the individual trips using, for instance, car pooling (hitchhike of people who work and live nearby) (9).

5. Reorganization/re-localization the mobility chain activities of the transport system in order to:

a. Reduce the average distance travelled in each trip: increasing the socio-economic activities density; regulating/influencing the companies and house localizations; and introducing new layouts for the urban development, oriented to public transport (namely car free zones, guaranteed land use for easy access to social and collective services, etc) (8);

b. Substitute the travel necessity, through work from home and internet shopping (although the latter is partially replaced by goods transportation to our homes) (9).

The intervention necessity starts at the strategically level of transport system and continues to the infrastructure project, through the environmental impact assessment, in order to include the environmental issues in the evaluation and selection of the best available options, when the system capacity is required to be reinforced (11) [7].

Subsequently, and based on the described above regarding sustainable mobility, three study-cases of the Almada (Portugal), Paris (France) and Barcelona (Spain) cities will be analyzed:

\subsection{Mobility action plan of Almada (Portugal)}

Almada, a Portuguese city located south of Lisbon and river Tejo, has a territorial area of $72 \mathrm{~km}^{2}, 35 \mathrm{~km}$ of coastline, with a total of 160825 inhabitants and a population density of $2000 \mathrm{hab} / \mathrm{km}^{2}$ (Censos 2001). Urban mobility is essentially done by car, through " 25 de Abril" bridge (about 160000 vehicles/day), to which high energy and environmental impacts are associated.

In this context, Almada's Energy Agency (AGENEAL) took action in two priority fields: Buildings (domestic and services) and Transports, which represent around $34 \%$ and $43 \%$ of energy consumption, respectively. Under these conditions and focusing on sustainable mobility, AGENEAL created a Sustainable Mobility Plan with the following objectives:

- Reduce car dependency on everyday journeys, increasing the number of trips by public transportation and climate-friendly means of transport;

- Improve public spaces and create comfort and safety conditions for pedestrians and cyclists. 
In order to achieve these objectives, AGENEAL developed a four stages plan:

1. Planning and developing a multimodal transportation system integrated in:

a. The Local Development Framework;

b. The Local Strategy for Climate Change;

c. The Mobility Plan, Accessibility 21;

d. The "Cyclable Almada" Plan;

e. The Local Strategy for Solidarity and Sustainable Development.

2. Creating infrastructures for Public Transportation and climate-friendly means of transport:

a. Tejo's South Metropolitan;

b. Almada Cyclable Network;

c. Traffic Control Measures.

3. Promoting new Technologies and Renewable Energies utilization:

a. Renewing city council fleet, according to energy efficiency criteria;

b. Optimization of urban solid waste collecting circuits;

c. Bicycle parking systems.

4. Informing, sensitizing and encouraging participation of citizens:

a. Trough forums to exchange views on major processes planning and in strategic studies Opinion Polls;

b. By campaign Promotion, thematic sessions and large outdoors initiatives [9].

\subsection{Mobility action plan of Paris (France)}

Paris, the capital of France, has 2153600 inhabitants with a population density of $24448 \mathrm{hab} / \mathrm{km}^{2}$ (2005). In face of the recent indicators of World Wildlife Foundation (WWF), the citizens of Paris have a footprint of about 3,0 hectares and according to a carbon audit 24,04 million $\mathrm{tCO}_{2}$ per year are emitted in Paris.

In reaction to the announced results, Paris assumed a compromise to do a Mobility Plan, a Climate Plan and to reduce $75 \%$ of greenhouse gases emissions.

The Mobility Plans are founded on a stage approach to fight the Climate Change and the pollution and to improve citizen's mobility based on:

- The reduction of $25 \%$ of traffic cars;

- The creation of 200 independent traffic lines (bicycles, cars and public transports) within seven years;

- Prioritizing public transports, with a raise of $12 \%$ in its utilization between 2001 and 2008;

- Prioritizing soft mobility with an increase of $70 \%$ generalized use of bicycle and an annual subscription of 215000 free bicycle users (Vélib's);

- The execution of economical activities easily in the urban zones;

- The improvement of air quality and on the decrease of greenhouse gases emissions with a reduction of $32 \%$ of $\mathrm{NO}_{\mathrm{x}}\left(-6 \%\right.$ in car traffic) and $9 \%$ of $\mathrm{CO}_{2}$ $(-11 \%$ in car traffic);

In the period of 2001-2020 the present Plan goals are more ambitious:

- $40 \%$ reduction of car traffic in Paris;

- 30\% increase of public transports offer;

- $19 \%$ increase of non motorized mobility ( $400 \%$ of bicycles); 
- $60 \%$ reduction of $\mathrm{CO}_{2}$ emissions (produced by traffic);

- $100 \%$ of Paris streets respecting the European Union directives relatively to $\mathrm{NO}_{2}$

- Improvement of road safety, noise and goods transportation [10].

\subsection{Mobility action plan of Barcelona (Spain)}

The Spanish city of Barcelona, capital of the Cataluña Autonomous Community, has a resident population of 1595110 inhabitants with a population density of 1 $523 \mathrm{hab} / \mathrm{km}^{2}$ (2006). Regarding to the mobility indicators Barcelona, 33,8\% of the inhabitants uses the private transport, $35,6 \%$ uses the public transport and $30,75 \%$ uses the soft mobility means of transportation.

The Barcelona Mobility Plan 2006-2012 is based on the following specific objectives:

- To increase the average vehicle occupation from 1,2 to 1,4 vehicles/person;

- To obtain an average emission of $120 \mathrm{~g} / \mathrm{km}$ of $\mathrm{CO}_{2}$ in all vehicles;

- To reduce in $6 \%$ the rate between vehicles $\mathrm{x} \mathrm{km} /$ day from current numbers;

- To increase in $30 \%$ the internal trips with public transports;

- To increase in $65 \%$ the internal and external trips.

The strategic objectives are related to the safety issues (reduction of accidents), to sustainable mobility (reduction of environmental impacts), to equity in mobility (accessible to all population), and to efficiency in mobility (reducing mobility to only the necessary, optimizing the excess).

The main actions of the Barcelona Mobility plan are presented below:

1. Safety on Mobility

a. Act on users behaviour;

b. Improve infrastructures and traffic management;

c. Carry through an inventory, an analysis and a precise assessment on accidents information.

2. Sustainable Mobility

a. Reduce greenhouse gases emissions;

b. Decrease atmospheric contamination;

c. Drop noise pollution.

3. Equity on Mobility

a. Guarantee a comfortable space for pedestrians and cyclists;

b. Improve the accessibilities for people with conditioned mobility

c. Increase the safety and autonomy of children;

d. Guarantee the right to mobility for population segments that do not have private vehicles (specially aged and young people) and others which do not use it in an abusive way.

4. Efficient Mobility

a. Reduce the necessary distances for displacement of the various services;

b. Optimize the private vehicle; 

c. Decrease of the displacement time, especially in the congestion
zones [11].

\section{Conclusions}

In the current conjuncture, the public transport assumes responsibility for about $40 \%$ of the total energy consumption in the European Union and it is accountable for $40 \%$ of $\mathrm{CO}_{2}$ emissions, which are the main cause of the Climate Change, as well as other impacts that degradate the quality of life of the population. It is understood that is urgent to adopt policies and measures that aim the prevention and mitigation of present effects of mobility. This, tends to become a sustainable mobility if has in sight the economic development (the preservation of work positions and the transference of goods, or access to services), the preservation of the environment (as long as it does not harm habitats and ecosystems, preserving the international commitments and agreements) and social (which guarantees the accessibility to all users, as the safety and equity on the distribution and use of the mobility agents).

From the analysis of the three Sustainable Mobility Plans of the three European cities, it can be concluded that:

- it does not exist a uniform indicator to evaluate the current state of mobility, because in some cases the sustainability indicator used is the "footprint", in others the "carbon footprint" or the final energy consumption or even the transport utilizations rates;

- is essential the adoption of reachable goals that go into European objectives, namely the "20-20-20 to 2020 " policy and international protocols, such as the Kyoto Protocol or others;

- The measures must be multifaceted and strong, involving all the community and adopting the best available technologies;

- It is important the existence of a network to share the information (EnergieCités, Forums, Research Centre's) in order to promote the best practices and to optimize the results;

- It is important the change of energy paradigms and the adjustment of behaviours, with push and pull measures, betting in the information and sensitization of the users.

The generalized adoption of Sustainable Mobility Plans will allow the cities to achieve the sustainable development.

\section{References}

[1] COM (97) 197 final, Para uma Agenda Urbana da União Europeia. Comissão das Comunidades Europeias, 6-05-1997, Bruxelas.

[2] Retrieved in $7^{\text {th }}$ September of 2009: http://ec.europa.eu/transport/ index_en.htm

[3] World Business Council for Sustainable Development, 2001

[4] Organisation de Coopération et Développement Économiques e Agence Internationale de L'Énergie 
[5] Alves, Mário J.; Mobilidade e acessibilidade: conceitos e novas práticas. Revista Indústria\&Ambiente, n. ${ }^{\circ}$ 55, March/April of 2009.

[6] Castanheira, Luís; Gouveia, B.; Energia, Ambiente e Desenvolvimento Sustentável. SPI, Porto, 2004.

[7] Moura, F.; Dimensões da mobilidade sustentável. Revista Indústria\&Ambiente, n. $^{\circ}$ 55, March/April of 2009.

[8] Report resumes "Sustainable European Cities" of the Expert group about Urban Environment. European Commission, Brussels, March of 1996.

[9] Freitas, C e Sousa, C.; Apresentação do Projecto BELIEF e os Casos Práticos de Almada na Área da Mobilidade, AGENEAL, Vila Nova de Gaia, $26^{\text {th }}$ of May of 2008 .

[10] Baupin, D; Paris Sustainable City.... Tokyo 's C40 conference, $22^{\text {nd }}$ of October of 2008

[11] Garcia, J.; Barcelona Urban Mobility Masterplan "2006 - 2012". Ajuntamento de Barcelona, June of 2009. 